\title{
On the space-charge boundary layer inside the nozzle of a cutting torch
}

\author{
L. Prevosto, ${ }^{1, a)}$ H. Kelly, ${ }^{2}$ and B. Mancinelli ${ }^{1}$ \\ ${ }^{1}$ Universidad Tecnológica Nacional, Regional Venado Tuerto, Las Heras 644, Venado Tuerto, Pcia. Santa \\ Fe 2600, Argentina \\ ${ }^{2}$ Departamento de Física, Instituto de Física del Plasma (CONICET), Facultad de Ciencias Exactas y \\ Naturales, UBA, Ciudad Universitaria Pab. I, Buenos Aires 1428, Argentina
}

(Received 4 March 2009; accepted 15 May 2009; published online 22 June 2009)

\begin{abstract}
A numerical study of the space-charge sheath adjacent to the nozzle wall of a cutting torch is presented. The hydrodynamic model corresponds to a collision-dominated sheath and does not assume cold ions, so drift-diffusion-type equations are used. Also an improved expression for the ion-neutral momentum transfer is employed rather than the usual constant ion-mean-free-path or constant ion collision frequency approximations. Assuming a constant electron temperature in the sheath and neglecting the electron inertial term, the continuity and momentum equations for ions and electrons, together with Poisson's equation, were solved for the electric potential, ion velocities (both normal and tangential components), and for the ion and electron densities. It was found that both the ion and electron densities present a sudden drop at the sheath-plasma edge. The ion density continues to decrease slowly inside the sheath, while the electron density presents a virtually zero value everywhere inside the sheath, the electron thermal conduction flux to the nozzle wall being negligible. These wall results thus become thermally isolated in spite of the high electron temperature in its adjacency. For a nozzle biasing voltage close to the gas breakdown, it was found that the electric field value is high, reaching a value of about $9 \times 10^{6} \mathrm{~V} \mathrm{~m}^{-1}$ at the exit of the nozzle wall. This value is higher than the average field value across the sheath and is on the order of the breakdown threshold value. This means that an undesired sheath breakdown could occur at the vicinities of the nozzle exit even if the average electric field across the sheath is not strong enough. (C) 2009 American Institute of Physics. [DOI: 10.1063/1.3153147]
\end{abstract}

\section{INTRODUCTION}

Plasma cutting torches produce a highly constricted, hot, and high velocity arc plasma jet between a cathode and a workpiece acting as the anode. That plasma is created by a narrow nozzle inside the torch, into which the gas is injected at a high pressure. ${ }^{1}$ Although there is a widespread industrial application of this technique, research efforts have appeared more recently in order to understand the main physical processes governing the phenomenon and to develop numerical models for the plasma-gas structure that could guide in advanced designs of cutting torches.

A classical diagnostic technique that has been employed to study cutting torches is charge collection through appropriately biased metallic conductors. This includes either electrostatic (Langmuir) probing ${ }^{2,3}$ or simply the nozzle surface. ${ }^{4}$ For a proper interpretation of the results, this kind of diagnostics requires knowledge on the non-neutral plasmaconductor boundary (sheath).

The problem of sheath formation at the plasma boundary is of importance for nearly all applications where the plasma is confined totally or partially to a finite volume by solid walls. ${ }^{5,6}$ For high-pressure thermal plasmas, local thermodynamic equilibrium (LTE), which usually holds in the plasma bulk, is violated near solid surfaces (walls or electrodes). In these regions the electron density is presumably much lower than $10^{23} \mathrm{~m}^{-3}$ (the lower limit for the existence of such equi-

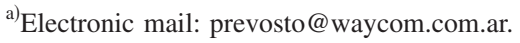

librium in a homogeneous plasma, according to Griem's criterion), and also very high temperature gradients may be present over the last few electron Debye lengths from the wall. ${ }^{4,7}$

When a plasma is in contact with a negatively biased surface (with a biasing voltage on the order or lower than the floating value), a strong electric field appears between the non-LTE (NLTE) plasma and that surface. This sheath becomes positively charged, rejecting electrons from the plasma and attracting ions to the negatively biased wall. The typical thickness of the sheath as compared to the characteristic lengths of the plasma (e.g., ion mean free path) determines the collisional degree of the sheath. Three regimes of sheath behavior can appear in high-pressure plasmas. There is a collision-dominated (i.e., mobility limited) regime when the sheath thickness is larger than the ion mean free path, a collisionless regime when the sheath is very thin, and a transition regime when both lengths are comparable. For the collision-dominated regime, expressions that describe the sheath are usually available for both cases of constant ion mean free path and constant ion mobility. ${ }^{8,9}$ In the opposite limit, when ion collisions are negligible, Child's law gives a simple description of the sheath. ${ }^{10}$ The number of ion mean free paths in the sheath needed to cause the transition from the collisionless to the collision-dominated regime for the constant mean-free-path model is only about one-half. ${ }^{11}$

Usually in high-pressure weakly ionized plasmas the sheath thickness is large compared to the ion mean free path, and the sheath is collision dominated. Such a picture corre- 


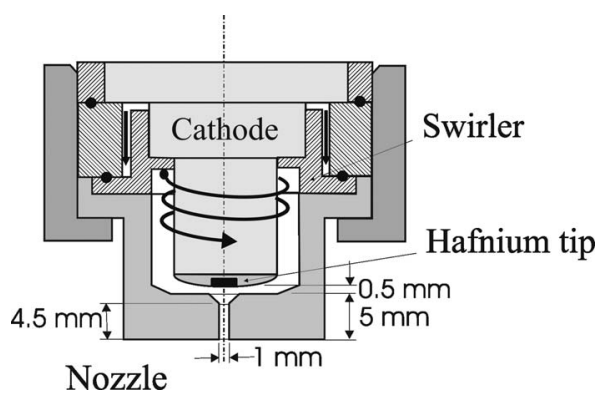

FIG. 1. Schematic of the arc torch indicating several nozzle geometric dimensions.

sponds to the space-charge sheath formed between the NLTE plasma and the nozzle wall inside a cutting torch. ${ }^{1-4,12-16}$ Near the plasma-sheath boundary, the electric field accelerating the ions toward the walls is negligible. Thus the fluid velocity of the ions is small compared to their thermal motion, and the collision frequency is independent of the ion fluid velocity. On the other hand, well in the sheath region, the electric field accelerates the ions to velocities comparable or larger than the thermal speed, and the collision frequency becomes proportional to the ion drift velocity. There is a smooth transition from a constant collision frequency of the ions within the plasma at the sheath edge to an approximately constant mean free path of the ions in the sheath regions close to the wall where high electric fields exist. The smooth transition between these two ion collision approximations appears where the potential drop over an ion mean free path becomes comparable to the ion thermal energy. ${ }^{17}$

The sheath-plasma problem in a collision-dominated regime was first studied in glow discharges, and most of the works ${ }^{6,8,9,11,18-22}$ considered cold ions, neglecting the ion pressure. The purpose of this paper is to present a collisiondominated model (ion mobility-limited motion) for the hydrodynamic description of the boundary space charge in contact with the nozzle wall inside a cutting torch. The model does not assume cold ions, so drift-diffusion-type equations are used. Also an improved expression for the ion-neutral momentum transfer ${ }^{17}$ is employed instead of the quoted ion collision approximations. The ion and electron densities, electrostatic potential, and ion velocity distributions are calculated inside the sheath. Boundary conditions for the numerical solutions within this sheath are based on the experimental plasma data previously obtained by the authors. ${ }^{4}$ In that work, the profiles of the sheath thickness, plasma density, and electron temperature at the sheath-plasma edge were obtained along the nozzle for measured values of the arc voltage and gas pressure.

The organization of this paper is as follows. In Sec. II the fluid equations of the collisional sheath model are presented. The results from the numerical solutions are presented and discussed in Sec. III, and in Sec. IV conclusion is derived.

\section{COLLISIONAL SHEATH MODEL}

A schematic of the arc torch indicating several nozzle geometric dimensions is presented in Fig. $1 .^{4}$ As it was shown in Refs. 6 and 19-22, when both plasma and sheath

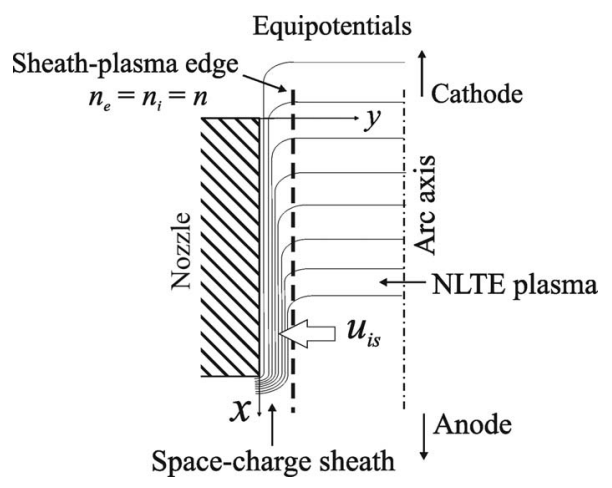

FIG. 2. Schematic of the sheath formed between the NLTE plasma and the nozzle wall.

are collisional, and the ionization inside the sheath can be neglected (so the sheath is source-free), there is a smooth joining between the plasma and the space-charge layer, thus avoiding the need of a transitional sheath (the presheath). So the sheath edge coincides with the unperturbed quasineutral plasma. The model geometry showing the collisional spacecharge sheath contiguous to the negatively biased nozzle is sketched in Fig. 2. Since the sheath remains thin as compared to the nozzle orifice radius, ${ }^{4}$ a planar geometry is used ( $y$ and $x$ are the normal and axial coordinates, see Fig. 2). At negative nozzle potentials (on the order or lower than the floating value), the electron density within the positive sheath remains small as compared to the ion density, so the sheath is considered source-free. The elastic mean free paths for all species are much smaller than the sheath thickness, and therefore the fluid description applies. Steady-state conditions are assumed.

The governing equations ${ }^{23}$ are given by the ion continuity equation,

$$
\nabla \cdot\left(n_{i} \bar{u}_{i}\right)=0
$$

the electron continuity equation,

$$
\nabla \cdot\left(n_{e} \bar{u}_{e}\right)=0,
$$

and the ion fluid momentum equation,

$$
n_{i} M\left(\bar{u}_{i} \cdot \nabla\right) \bar{u}_{i}=-\nabla\left(n_{i} k T_{h}\right)-e n_{i} \nabla \phi+n_{i} M\left(\bar{u}_{n}-\bar{u}_{i}\right) \nu_{i},
$$

where $\bar{u}_{e}$ is the electron fluid velocity and $n_{e}$ is the electron density. $\bar{u}_{i}, n_{i}$, and $M$ are the fluid velocity, density, and mass of the ions, respectively. $k$ is Boltzmann's constant and $T_{h}$ is the heavy particle temperature (ions and neutrals). $\phi$ is the electrostatic potential and $e$ is the electron charge. The last term in Eq. (3) represents the drag force due to the collisions between ions and neutrals. $\bar{u}_{n}$ is the neutral fluid velocity and $\nu_{i}$ is the ion-neutral collision frequency for momentum transfer.

The electron fluid momentum equation is

$$
n_{e} m\left(\bar{u}_{e} \cdot \nabla\right) \bar{u}_{e}=-\nabla\left(n_{e} k T_{e}\right)+e n_{e} \nabla \phi-n_{e} m \bar{u}_{e} \nu_{m},
$$

where $m$ and $T_{e}$ are the mass and temperature of the electrons, respectively. $\nu_{m}$ is the effective collision frequency for momentum transfer. Finally, Poisson's equation relates the 
difference between ion and electron densities within the sheath to the self-consistent potential,

$$
\varepsilon_{0} \nabla^{2} \phi=-e\left(n_{i}-n_{e}\right),
$$

where $\varepsilon_{0}$ is the vacuum permittivity.

The inertial term can be usually dropped from Eq. (4) because of the smallness of the electron mass. Then, considering the Einstein relation, Eq. (4) reduces to the total electron flux, ${ }^{10}$

$$
\bar{\Gamma}_{e} \equiv n_{e} \bar{u}_{e}=n_{e} \mu_{e} \nabla \phi-D_{e} \nabla n_{e},
$$

where the usually small thermodiffusion term (proportional to $-\nabla T_{e}$ ) was neglected in Eq. (4) as compared to the diffusion term. ${ }^{10} \mu_{e}$ and $D_{e}$ are the electron mobility and diffusion coefficients, respectively. If the nozzle potential is sufficiently negative due to the high mobility of the electrons, oppositely directed high diffusion and drift electron fluxes approximately balance each other to yield a small resultant total electron flux comparable to (or less than) the ion flux. Hence $\bar{\Gamma}_{e} \approx 0$ in Eq. (6) and the electron density inside the sheath obeys the relation, ${ }^{10}$

$$
n_{e}=n \exp \left[\frac{e\left(\phi-\phi_{s}\right)}{k T_{e}}\right],
$$

where $n \equiv n_{i} \cong n_{e}$ and $\phi_{s}$ are the density and the electrostatic potential of the plasma at the sheath-plasma edge, respectively. The neutral particles are considered at rest (i.e., $u_{i}$ $\left.\gg u_{n}\right)$. To close the model, an expression for the ion momentum transfer by elastic collisions must be established. Two special cases are usually treated in literatures: ${ }^{11,17,18}$ constant ion mean free path and constant ion collision frequency. In the first case, the basic assumption is $e|\nabla \phi| \lambda_{i} \gg k T_{h}$, so the ion drift velocity is much larger than the ion thermal velocity. Hence the drag force is modeled by $-M u_{i} \bar{u} / \lambda_{i}$, where the ion mean free path $\lambda_{i} \equiv\left(n_{n} \sigma\right)^{-1}$ is constant $\left(n_{n}\right.$ is the neutral gas density and $\sigma$ is the momentum transfer cross section for elastic collisions between ions and neutrals). The collision frequency $\nu_{i}=u_{i} / \lambda_{i}$ depends, in this case, on the ion fluid velocity. In the opposite limit, the assumption $e|\nabla \phi| \lambda_{i}$ $\ll k T_{h}$ applies. With this condition satisfied, the ion fluid velocity is much smaller than its thermal speed. The collision frequency of the ions is thus determined by their random thermal motion rather than their fluid velocity and thus $\nu_{i}$ $=\sqrt{2} u_{\mathrm{th}} / \lambda_{i}$. In this relation, the ion thermal velocity is given as $u_{\mathrm{th}}=\sqrt{8 k T_{i} /(\pi M)}$, and the constant ion collision frequency is independent of the fluid velocity. The factor $\sqrt{2}$ is due to the mutual motion of the ions and neutral assuming the same temperature for both species. ${ }^{7}$ The drag force in this case is given as $-M \nu_{i} \bar{u}_{i}$. Both physical approximations assume that the collision cross section is independent of the ion fluid velocity $\left(\approx 5 \times 10^{-19} \mathrm{~m}^{2}\right){ }^{24}$ At high pressures, for strongly collisional sheaths, the constant ion mean-free-path approximation applies close to the wall where the electric field strength is stronger. On the other hand, the constant ion mobility approximation (constant ion collision frequency) is physically more accurate at the sheath-plasma edge (where the electric field is relatively weak). In the transition region, the collision frequency is given by $\nu_{i}\left(u_{i}\right)=\sqrt{\left(\nu_{\lambda}\right)^{2}+\left(\nu_{\nu}\right)^{2}}, 17$ where $\nu_{\lambda}$ and $\nu_{\nu}$ are the ion collision frequencies in the quoted previous approximations. Following this approach, the ion collision frequency can be written as

$$
\nu_{i}\left(u_{i}\right)=\frac{\sqrt{u_{i}^{2}+2 u_{\mathrm{th}}^{2}}}{\lambda_{i}} .
$$

In spite of the collisional nature of the sheath, inelastic electron collisions are very rare and also the electron energy transfer to heavy particles by elastic collisions is small. Therefore, it was assumed that $T_{e} \approx$ const inside the sheath with a value corresponding to the sheath-plasma edge value.

The model is now closed. In the limit of strong ionneutral collisions (i.e., the mobility-limited ion motion approximation), the collision parameter $D / \lambda_{i}$ is large ( $D$ is the sheath thickness). Using the data obtained in our previous work, ${ }^{4} D / \lambda_{i} \approx 10^{2}$. In such circumstances, Eq. (3) is simplified by neglecting the convective term on its left hand side. Combining Eqs. (1), (3), (5), (7), and (8), a system of coupled partial differential equations for describing the mobility-limited ion collisional sheath is obtained,

$$
\begin{aligned}
& \frac{\partial\left(n_{i} u_{i x}\right)}{\partial x}+\frac{\partial\left(n_{i} u_{i y}\right)}{\partial y}=0, \\
& -\frac{\partial\left(n_{i} k T_{h}\right)}{\partial x}-e n_{i} \frac{\partial \phi}{\partial x}-n_{i} M u_{i x} \nu_{i}=0, \\
& -\frac{\partial\left(n_{i} k T_{h}\right)}{\partial y}-e n_{i} \frac{\partial \phi}{\partial y}-n_{i} M u_{i y} \nu_{i}=0, \\
& \frac{\partial^{2} \phi}{\partial x^{2}}+\frac{\partial^{2} \phi}{\partial y^{2}}=-\frac{e}{\varepsilon_{0}} n\left[\frac{n_{i}}{n}-\exp \left(\frac{e\left(\phi-\phi_{s}\right)}{k T_{e}}\right)\right],
\end{aligned}
$$

where the ion collision frequency is given by Eq. (8). A similar plasma-sheath model was presented in Ref. 11 for a two-fluid ( $T_{h} \ll T_{e}$, i.e., cold ions) uniform plasma but under the above quoted extreme collisional approximations. The present model is further complicated by the axial potential drop along the arc column facing the equipotential nozzle (see Fig. 2). Also for large ion temperatures (in this problem, $T_{i}$ is comparable to $T_{e}$ ), the thermal ion flux to the wall cannot be neglected; therefore, the diffusive term in Eq. (3) must be considered.

To solve Eqs. (8)-(12), appropriate boundary conditions must be specified. In doing this, the profiles of the sheath thickness, plasma density, and electron temperature at the sheath-plasma edge, arc voltage, and gas pressure inside the nozzle were taken from Ref. 4. For instance, a typical $n$ profile at the sheath-plasma edge is presented in Fig. 3. At the nozzle wall $(y=0)$, the voltage of the nozzle is known $\left(\phi_{N}\right)$. The sheath-plasma edge $(y=D)$ coincides with the quasineutral plasma, so $n \equiv n_{i} \cong n_{e}$ and the voltage distribution is the arc (variable) voltage $\left[\phi_{\text {arc }}(x)\right]$. Also the radial electric field value at the sheath-plasma edge is very small, ${ }^{10}$ hence $(\partial \phi / \partial y)_{y=D} \approx 0$. The ions enter the sheath from the plasma with a velocity normal to the boundary surface given by $u_{i s} \approx u_{B}\left(\lambda_{i} / \lambda_{D s}\right)^{1 / 2}$, ${ }^{22}$ where $u_{B} \equiv \sqrt{k T_{e} / M}$ is the Bohm velocity and $\lambda_{D s}$ the electron Debye length at the sheath entrance. At the nozzle inlet $(x=0)$ and exit $\left(x=L_{n}\right)$, open 


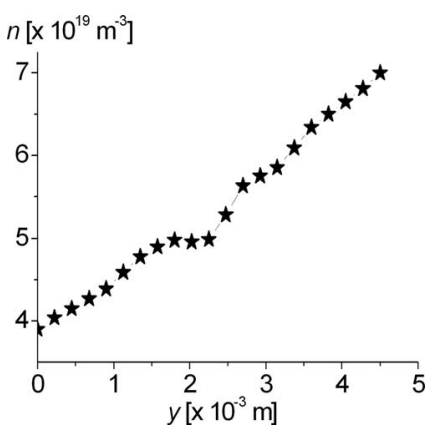

FIG. 3. (Color online) Plasma density profile at the plasma-sheath edge, reprinted with permission from L. Prevosto, H. Kelly, and B. Mancinelli, J. Appl. Phys. 105, 013309 (2009). Copyright 2009, American Institute of Physics.

boundary conditions are assumed. So the quantities $\partial u_{i x} / \partial x$, $\partial u_{i y} / \partial x, \partial n_{i} / \partial x$, and $\partial \phi / \partial x$ are conserved through these surfaces.

\section{NUMERICAL RESULTS AND DISCUSSION}

The governing Eqs. (8)-(12) were solved for the electric potential, ion velocity (both components), and ion density by integrating them numerically using a finite difference discretization technique in a $100 \times 50$ uniform grid. An iterative method was adopted, which continued until the solutions of the physical quantities reached the following criterion:

$$
\frac{\chi^{(n)}-\chi^{(n-1)}}{\chi^{(n)}} \leq 10^{-5}
$$

with $\chi^{(n)}$ being the $n$ iteration of a generic variable $\chi$. This convergence criterion was found to be sufficient since negligible differences in the final solutions were found when lower values for the convergence criterion were used.

For a $30 \mathrm{~A}$ arc current and an oxygen gas mass flow of $0.32 \mathrm{~g} \mathrm{~s}^{-1}$ and $\phi_{n}=-153 \mathrm{~V}$, a thin sheath with an almost constant thickness of $D(z)=21 \mu \mathrm{m}$ (see Fig. 4 of Ref. 4) was formed between the plasma and the nozzle wall. The resulting value of $T_{e}$ in these conditions was $T_{e}=5400 \mathrm{~K}$. It should be noted that the quoted nozzle bias potential value is close to a gas breakdown situation (double arcing) in this torch. This situation was chosen to look for a connection (if any) between the undesired breakdown and some characteristic of the sheath structure.

Linear distributions of the arc voltage and pressure $(p)$ inside the nozzle were assumed as in Ref. 4, $\phi_{\text {arc }}(x)$ $=\phi_{\text {arc }}(0)+x\left[\phi_{\text {arc }}\left(L_{n}\right)-\phi_{\text {arc }}(0)\right] / L_{n}$ and $p(x)=p(0)+x\left[p\left(L_{n}\right)\right.$ $-p(0)] / L_{n}$, where $p(0)=0.55 \mathrm{MPa}, \phi_{\mathrm{arc}}(0)=-70 \mathrm{~V}, p\left(L_{n}\right)$ $=0.10 \mathrm{MPa}$, and $\phi_{\text {arc }}\left(L_{n}\right)=-22 \mathrm{~V}$. Since the plasma density is very low, close to the wall, the neutral gas density can be immediately obtained from the state equation with the assumed values of the neutral temperature value close to the wall $\left(T_{h}=1000 \mathrm{~K}\right)($ Ref. 4$)$ and the $p$ value (neglecting the plasma pressure). Then the neutral density is given by $n_{n}$ $=p /\left(k T_{h}\right)$. The ion velocity at the sheath entrance $u_{B}$ $\equiv \sqrt{k T_{e} / M}\left(n_{n} \sigma_{0} \lambda_{D s}\right)^{-1 / 2}$ was also calculated taking into account the $T_{e}$ values at the sheath-plasma edge (from Ref. 4) and $p$.

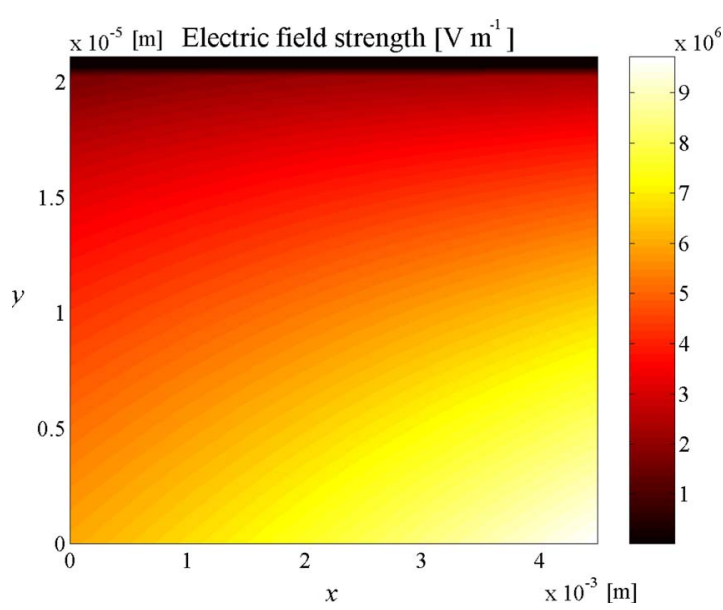

FIG. 4. (Color online) Spatial distribution of the electric field strength inside the sheath.

In Fig. 4, the spatial distribution of the electric field strength is presented. As it can be seen, the electric field value is high, with the largest values along the nozzle wall, varying from $6 \times 10^{6} \mathrm{~V} \mathrm{~m}^{-1}$ near the nozzle entrance to about $9 \times 10^{6} \mathrm{~V} \mathrm{~m}^{-1}$ at the nozzle exit. This last value is higher than the average field value across the sheath and is on the order of the breakdown threshold value. This means that an undesired sheath breakdown could occur close to the nozzle exit even if the average electric field across the sheath is not strong enough.

The spatial distributions of the ion and electron densities inside the sheath are presented in Figs. 5 and 6. As it can be seen in these figures, $n_{i}$ drops sharply near the sheath edge and continues to decrease slowly, while the electron density also shows a very steep drop near the sheath edge, with a virtually zero value everywhere inside the sheath. The lack of electrons inside the sheath implies that the electron thermal conduction flux to the nozzle wall can be neglected. The nozzle wall results thus become thermally isolated in spite of the high electron temperature in its adjacency.

It was found that both ion and electron densities decrease when the electrostatic potential decreases. This behavior of $n_{i}$ is due to the ion acceleration at an almost constant

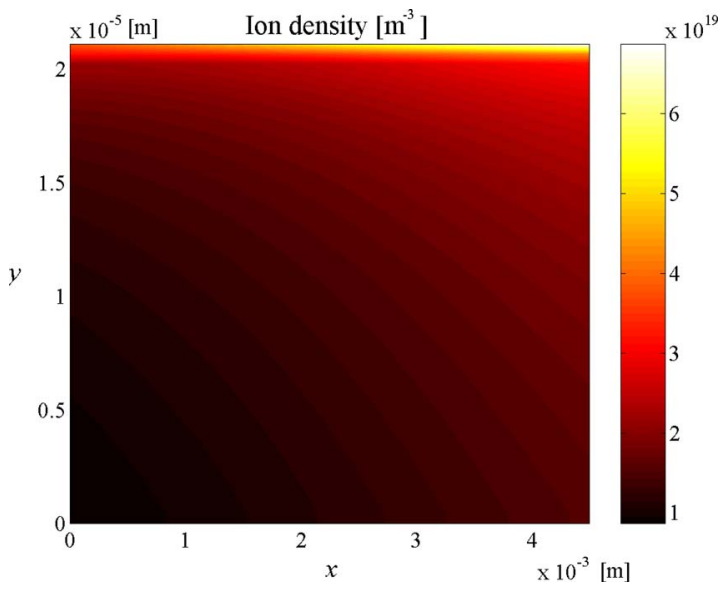

FIG. 5. (Color online) Spatial distribution of the ion density inside the sheath. 


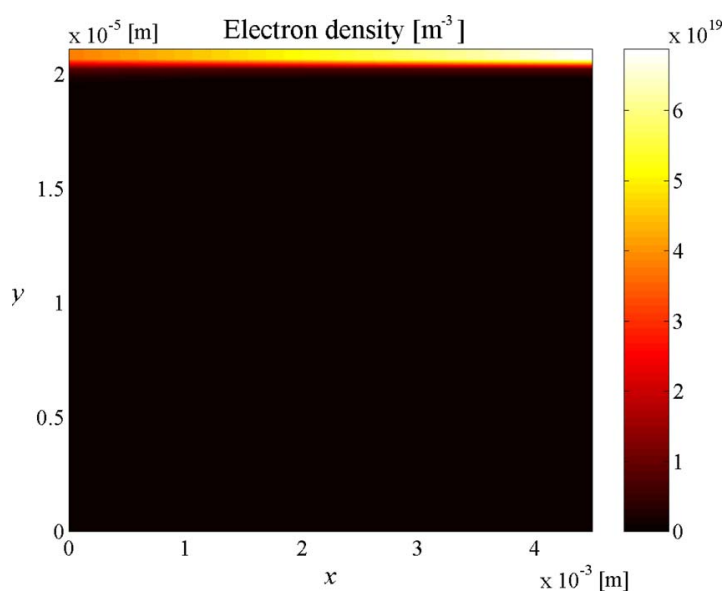

FIG. 6. (Color online) Spatial distribution of the electron density inside the sheath.

ion flux, while the $n_{e}$ behavior is due to the fact that the electrons are related to the electric field according to Boltzmann's equation [Eq. (7)].

\section{CONCLUSIONS}

A numerical study of the space-charge sheath in contact with the nozzle wall of a cutting torch has been presented. The hydrodynamic model corresponds to a collisiondominated sheath and does not assume cold ions, so driftdiffusion-type equations are used (instead of low-power, low-ionization degree discharges). Also an improved expression for the ion-neutral momentum transfer is employed rather than the usual constant ion mean-free-path or constant ion collision frequency approximations. Assuming a constant electron temperature in the sheath and neglecting the electron inertial term, the continuity and momentum equations for ions and electrons, together with Poisson's equation, were solved for the electric potential, ion velocities (both normal and tangential components), and ion and electron densities. The equations were numerically integrated using a finite difference discretization technique in a $100 \times 50$ uniform grid. Boundary conditions for the numerical solutions were based on the experimental plasma data previously obtained $^{4}$ for the plasma region adjacent to the nozzle wall. In that work, the profiles of the sheath thickness, plasma density, and electron temperature at the sheath-plasma edge, and arc voltage, and gas pressure along the nozzle were derived.

The obtained profiles of the ion and electron densities inside the sheath are in good agreement with what is expected for every plasma-sheath surrounding a negatively biased solid body immersed in plasma and explain why a copper nozzle with a relatively low melting temperature can be thermally isolated from the hot plasma by such a thin layer of gas: the absence of electrons in the sheath makes the electron thermal conduction flux to the nozzle wall negligible.

The detailed calculation of the electric field values inside the sheath allows predicting the possibility of a gas breakdown (according to Pashen's law ${ }^{10}$ ) in the region adjacent to the nozzle. This eventual breakdown leads to double arcing, which is known to be one of the largest drawbacks of cutting torches. Moreover, the model results show that local values of the electric field can be considerably larger than the average field value across the sheath, mainly close to the nozzle exit. This means that an undesired sheath breakdown could occur at the nearness of the nozzle exit even if the average electric field across the sheath is not strong enough.

\section{ACKNOWLEDGMENTS}

This work was supported by grants from the Universidad de Buenos Aires (Grant No. PID X111), CONICET (Grant No. PIP 5378), and UTN (Grant No. PID Z 012). One of the authors (L.P.) is grateful to the Fundación YPF. Also B.M. is grateful to the Universidad Tecnológica Nacional. H.K. is a member of the CONICET.

${ }^{1}$ V. A. Nemchinsky and W. S. Severance, J. Phys. D: Appl. Phys. 39, R423 (2006).

${ }^{2}$ L. Prevosto, H. Kelly, and B. Mancinelli, IEEE Trans. Plasma Sci. 36, 263 (2008).

${ }^{3}$ L. Prevosto, H. Kelly, and B. Mancinelli, IEEE Trans. Plasma Sci. 36, 271 (2008).

${ }^{4}$ L. Prevosto, H. Kelly, and B. Mancinelli, J. Appl. Phys. 105, 013309 (2009).

${ }^{5}$ K. U. Riemann, J. Phys. D: Appl. Phys. 24, 493 (1991).

${ }^{6}$ R. N. Franklin, J. Phys. D: Appl. Phys. 36, R309 (2003).

${ }^{7}$ M. Boulos, P. Fauchais, and E. Pfender, Thermal Plasmas, Fundamentals and Applications (Plenum, New York, 1994), Vol. 1.

${ }^{8}$ R. N. Franklin, J. Phys. D: Appl. Phys. 35, 2270 (2002).

${ }^{9}$ K. U. Riemann, J. Phys. D: Appl. Phys. 36, 2811 (2003).

${ }^{10}$ Y. P. Raizer, Gas Discharge Physics (Springer, Berlin, 1991)

${ }^{11}$ T. E. Sheridan and J. Goree, Phys. Fluids B 3, 2796 (1991).

${ }^{12}$ L. Girard, Ph. Teulet, M. Razafinimanana, A. Gleizes, F. Camy-Peyret, E. Baillot, and F. Richard, J. Phys. D: Appl. Phys. 39, 1543 (2006).

${ }^{13}$ P. Freton, J. J. Gonzalez, A. Gleizes, F. Camy Peyret, G. Caillibotte, and M. Delzenne, J. Phys. D: Appl. Phys. 35, 115 (2002).

${ }^{14}$ P. Freton, J. J. Gonzalez, F. Camy Peyret, and A. Gleizes, J. Phys. D: Appl. Phys. 36, 1269 (2003).

${ }^{15}$ C. Pardo, J. González-Aguilar, A. Rodríguez Yunta, and M. A. G. Calderón, J. Phys. D: Appl. Phys. 32, 2181 (1999).

${ }^{16}$ J. Peters, J. Heberlein, and J. Lindsay, J. Phys. D: Appl. Phys. 40, 3960 (2007).

${ }^{17}$ Z. Sternovsky and S. Robertson, IEEE Trans. Plasma Sci. 34, 850 (2006).

${ }^{18}$ T. E. Sheridan and M. J. Goeckner, J. Appl. Phys. 77, 4967 (1995).

${ }^{19}$ J. L. Blank, Phys. Fluids 11, 1686 (1968).

${ }^{20}$ R. N. Franklin, J. Phys. D: Appl. Phys. 37, 1342 (2004).

${ }^{21}$ R. N. Franklin, J. Phys. D: Appl. Phys. 36, 2821 (2003).

${ }^{22}$ R. N. Franklin, IEEE Trans. Plasma Sci. 30, 352 (2002).

${ }^{23}$ R. J. Goldston and P. H. Rutherford, Introduction to Plasma Physics (Institute of Physics, Bristol, 1995).

${ }^{24}$ H. L. Anderson, A Physicist's Desk Reference, 2nd ed. (AIP, New York, 1989). 